\title{
Performance Comparison between MAI and Noise Constrained LMS Algorithm for MIMO CDMA DFE and Linear Equalizers
}

\author{
Khalid Mahmood \\ Member Institute of Electrical and Electronic Engineers (IEEE)
}

\begin{abstract}
This paper presents a performance comparison between a constrained least mean squared algorithm for MIMO CDMA decision feedback equalizer and linear equalizer. Both algorithms are constrained on the length of spreading sequence, number of users, variance of multiple access interference as well as additive white Gaussian noise (new constraint). An important feature of both algorithms is that multiple access interference together with noise variance is used as a constraint in MIMO CDMA linear and decision feedback equalization systems. Convergence analysis is performed for algorithm in both cases. From the simulation results shown at the end show that algorithm developed for decision feedback equalizer has outperformed the algorithm developed for linear equalizer in MIMO CDMA case
\end{abstract}

Keywords-Least mean squared algorithm (LMS); linear equalizer (LE); multiple input; multiple output (MIMO); decision feedback equalizer (DFE); multiple access interference (MAI); Variance; adaptive algorithm

\section{INTRODUCTION}

It is shown in literature that performance of an adaptive algorithm may be enhanced if partial knowledge of the channel is included in algorithm design[1], [2], [3]. By using this idea, [1] developed an algorithm called noise-constrained least mean squared (LMS) for tracing of finite impulse response (FIR) channels by utilizing the statistics of the additive noise. An important feature of this algorithm is its superiority over traditional LMS algorithm in convergence while having almost same computational complexity. A complementary pair LMS (CP-LMS) [4], [5] was introduced later on using a constrained optimization technique named augmented Lagrangian. This technique could be used to resolve the problem of selecting an appropriate update step-size in LMS algorithm. Augmented technique was used in [6], incorporating the knowledge of the statistics (variance) of multiple access interference (MAI) and additive white noise and was named constrained LMS algorithm (MNCLMS) for single input, single ouput (SISO) CDMA system.

Since the MAI together with the white Gaussian noise (AWGN) badly effects the MIMO-CDMA systems, it is required to design a receiver design that would negate the damaging effect of MAI and additive AWGN. This necessitates an enactment of the MNCLMS algorithm derived in [7], [8] for the decision feedback equalizer (DFE) case. The said algorithm is developed by including statistics such as MAI and noise variances and is named MIMO-MAI plus noise constrained LMS (MIMO-MNCLMS) adaptive algorithm. A MIMO implementation of the MAI and noise constrained algorithm for linear equalizer (LE) was developed in [9]. In this paper, we have presented the performance comparison of the MNCLS algorthm in decesion feedbacj equalizer (DFE) and linear equalizer (LE).

Our paper is presented as,

Introductory remarks are given in section I, Section II discusses system model. Algorithm motivation for LE and DFE are presented in section III, whereas section $\mathrm{V}$ deals with computational complexity of the algorithm. Section VI presents the performance comparison between the DFE and LE for MNCLS algorithm. Conclusion is provided in section VII.

\section{SySTEM MODEL}

In this paper, a typical CDMA transmitter model for a downlink of a mobile radio network is considered as shown in Fig. 1. It comprises of $N$ transmitters and $M$ receivers.

In this paper, we are using a fast Rayleigh fading channel. The impulse response between the $n$th transmitter and $m$ th receiver for an $l$ th symbol is [9]

$$
H_{m n}^{l}(t)=h_{m n}^{l} e^{j \phi_{l}} \delta(t)
$$

where

$h_{m n}^{l}$ is the impulse response

$\phi_{l}$ is the phase of Rayleigh channel.

The sensor in the $m$ th receiver sees the following,

$$
\begin{aligned}
r_{m}(t)= & \sum_{n=1}^{N} \sum_{l=-\infty}^{\infty} \sum_{k=1}^{K} A^{k} b_{n}^{l, k} s_{n}^{l, k}(t) h_{m n}^{l} \\
& +\nu_{m}(t), \quad m=1,2, \ldots M
\end{aligned}
$$

where

$K=$ represents number of users,

$s_{n}^{l, k}(t)=$ represents rectangular signature waveform

$\left\{b_{n}^{l, k}\right\}=$ input bit stream of the $k$ th user,

$h_{m n}^{l}=$ channel tap between the $m$ th transmiting antenna and the $n$th receiving antenna,

$A^{k}=k$ th user amplitude,

$\varpi_{m}=$ additive AWGN. It has zero mean 


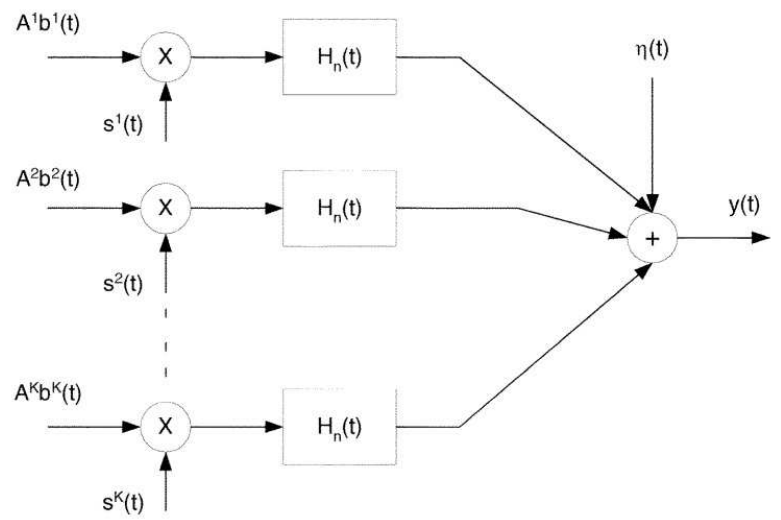

Fig. 1. Synchronous downlink CDMA system model

The receiving side is comprised of a matched filter. It is matched to the signature waveform of user 1 (desired user). as:

Output of the matched filter at the $m$ th receiver is set up

$$
\begin{aligned}
y_{m}^{l}= & \int_{(l-1) T_{b}}^{l T_{b}} r_{m}(t) s_{m}^{l, 1}(t) d t \\
= & \sum_{n=1}^{N} A^{1} b_{n}^{l, 1} h_{m n}^{l}+\sum_{n=1}^{N} \sum_{k=2}^{K} A^{k} b_{n}^{l, k} \rho_{n}^{k, 1}(t) h_{m n}^{l} \\
& +\varpi_{m}, \quad m=1,2, \ldots M
\end{aligned}
$$

MAI is represented by the Second term in equation 3. Mathematically, it is defined as:

$$
\begin{aligned}
z_{m}^{l} & =\sum_{n=1}^{N} \sum_{k=2}^{K} A^{k} b_{n}^{l, k} \rho_{n}^{k, 1} h_{m n}^{l}, \quad m \\
& =1,2, \ldots M
\end{aligned}
$$

MAI may also be set up as

$$
\begin{aligned}
z_{m}^{l} & \leq \sum_{n=1}^{N} \sum_{k=2}^{K} A^{k} b_{n}^{l, k} \rho_{n}^{k, 1} \sum_{n=1}^{N} h_{m n}^{l} \\
& \leq U_{m} \sum_{n=1}^{N} h_{m n}^{l}, \quad m=1,2, \ldots M
\end{aligned}
$$

where,

$$
U_{m}^{l}=\sum_{n=1}^{N} \sum_{k=2}^{K} A^{k} b_{n}^{l, k} \rho_{n}^{k, 1} .
$$

\section{Algorithm Motivation}

Certain adaptive algorithms (LMS and recursive least square (RLS)) don't use models for channel coefficients and additive noise, on the other hand, model based algorithms (random walk, auto-regressive etc) use models for estimating channel coefficients and AWGN [10].

Performance of an adaptive algorithm may be enhanced if even fractional knowledge of a channel statistics is available. According to the noise constrained LMS algorithm [3], weight update equation of an algorithm can be set up as

$$
\mathbf{w}_{n+1}=\mathbf{w}_{n}+\mu_{n}^{l} e_{n} \mathbf{X}_{n}^{l}
$$

where

$\mathbf{X}_{\mathbf{n}}^{\mathbf{l}}=$ the input data

$\mu_{n}^{l}=$ positive step size

$e_{n}^{l}=$ error between the output of the matched filter and an adaptive filter

$$
\mu_{n+1}=2 \mu_{n}^{l}\left(1+\gamma \lambda_{n}\right)
$$

$$
\lambda_{n+1}=\lambda_{n}+\beta\left[\left(\frac{1}{2} e_{n}^{2}-\sigma_{\nu_{m}^{l}}^{2}\right)-\lambda_{n}\right]
$$

Where $\lambda, \alpha$ and $\beta$ are positive step sizes (parameters). Computational cost of above mentioned algorithm is same as LMS but the convergence rate of the algorithm is much better than the LMS.

As MAI is a major factor in the system performance of a multiuser environment, it becomes important to come up with a receiver scheme which would reduce the damaging effects introduced by MAI and AWGN. In the previous research work, MAI was assumed to be a part of AWGN which is not valid. But using MAI alone as a constraint is not a viable choice since noise is an undeniable physical constraint and may not be ignored while developing such algorithms.

As NCLMS algorithm is noise constrained only, a new constrained algorithm is established for LE and DFE in [9] and [8] respectively. Variance of MAI and AWGN was used as new constraints in these algorithms.

\section{MIMO-CDMA MAI AND NOISE CONSTRAINED LMS ALGORITHM}

\section{A. MIMO-CDMA MNCLMS Constrained Algorithm for Lin-} ear Equalizer (LE)

LE algorithm was developed in [9] and is given as

$$
\mathbf{s}_{n}^{l+1}=\mathbf{s}_{n}^{l}+\varsigma_{n}^{l} e_{n}^{l} E_{n}^{l}
$$

where

$\varsigma_{n}^{l}=$ positive step size and is is the positive step size,

$e_{n}^{l}=$ error of output of a matched filter and an adaptive filter. It is mathematiclly defined as:

$$
e_{n}^{l}=\hat{x}_{n}-\mathbf{s}_{n}^{l} E_{n}^{l}
$$

$E_{n}^{l}$ in equation 10 is a joint input to the LE and is:

$$
E_{n}^{l}=\left[\left(\mathbf{y}_{n}^{l}\right)^{T}\right]
$$

Order of $E_{n}^{l}=(M L \times 1)$. 


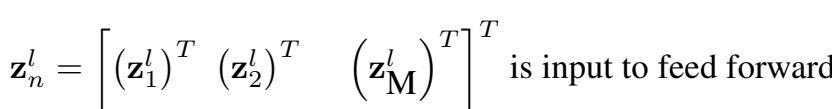
filter $(\mathrm{FFF})$ having dimension $M L \times 1$. It is a collection of vectors consisting of $z_{m}^{l}$ given by $\mathbf{z}_{m}^{l}=\left[\begin{array}{lll}z_{m}^{l} & z_{m}^{l-1} & z_{m}^{l-L+1}\end{array}\right]^{T}$.

and

$$
\begin{aligned}
\hat{\mathbf{x}}_{n} & =\mathbf{w}_{o}^{T} E_{n}^{l} \\
& =\mathbf{x}_{n}^{l}+\bar{\varphi}_{n}^{l}
\end{aligned}
$$

or

$$
\mathbf{x}_{n}^{l}=\mathbf{w}_{o}^{T} E_{n}^{l}-\bar{\varphi}_{n}^{l}
$$

$\bar{\varphi}_{n}^{l}$ is a filtered noise passing through (FFF). It is comprised of MAI and AWGN.

$\varsigma_{n}^{l}$, is shown to be

$$
\begin{gathered}
\varsigma_{n}^{l}=\varsigma_{n}\left(1+\gamma_{n} \lambda_{n}^{l}\right) m \\
=1,2, \ldots, M \\
\lambda_{n}^{l+1}=\lambda_{n}+\beta_{n}\left[\frac{1}{2}\left(e_{n}^{l^{2}}-\sigma_{\bar{\varphi}_{n}^{l}}^{2}\right)-\lambda_{n}^{l}\right] m \\
=1,2, \ldots, M
\end{gathered}
$$

where,

$\sigma_{\bar{\varphi}_{n}^{l}}^{2}$ is variance of the filtered noise.

B. MIMO-CDMA MNCLMS Constrained Algorithm for Decision Feedback Equalizer (DFE)

MIMO-CDMA MNCLMS algorithm was developed in [11] and is given as

$$
\mathbf{w}_{n}^{l+1}=\mathbf{w}_{n}^{l}+\mu_{n}^{l} e_{n}^{l} E_{n}^{l}
$$

where,

$E_{n}^{l}$ is the combined input to the DFE and is given by

$$
E_{n}^{l}=\left[\begin{array}{ll}
\left(\mathbf{y}_{n}^{l}\right)^{T} & \left(\mathbf{x}_{n}^{l}\right)^{T}
\end{array}\right]^{T}
$$

and is of the order of $(M K+N Q) \times 1)$

$\mu_{n}^{l}$ is a positive step size and is

$$
\begin{gathered}
\mu_{n}^{l}=\mu_{n}\left(1+\gamma_{n} \lambda_{n}^{l}\right) m \\
=1,2, \ldots, M \\
\lambda_{n}^{l+1}=\lambda_{n}+\beta_{n}\left[\frac{1}{2}\left(e_{n}^{l^{2}}-\sigma_{\overline{\nu_{n}^{l}}}^{2}\right)-\lambda_{n}^{l}\right] m \\
=1,2, \ldots, M
\end{gathered}
$$

\section{v. Computational Cost of LE And DFE Algorithms}

Computational cost of any algorithm is an important aspect of that algorithm. Increased complexity can reduce the effectiveness of an algorithm. A tradeoff between performance and computational complexity is possible if increased complexity results in substantial performance gains. In this section, we are comparing computational costs of few algorithms. As shown in tables I and II, computational cost of the algorithm in case of DFE is more than the LE but cost is much lower than the RLS. Computational cost of the DFE and the LE algorithms is more than [6] but that is for SISO CDMA case, whereas, algorithms developed in [9] and [11] are for MIMO CDMA case.

\section{Simulation Results}

Following independent assumptions are used while performing the comparison analysis [13], [14].

1) Input random process $\left\{\mathbf{x}_{n}^{l}\right\}$ is an independent and identically distributed (i.i.d) random proces.

2) AWGN is a zero mean i.i.d, Gaussian random process and is independent of input process.

3) MAI in AWGN environment is zero mean Gaussian random process. It is independent of the input process as well as AWGN.

\section{A. Interference Elimination in an AWGN Channel for LE and DFE cases}

In oreder to analyze the performance of the proposed algorithm for MIMO CDMA LE case, simulation results are presented in this section. Performance of MNCLMS algorithm is compared to standard LMS, MCLMS noise constrained LMS and zero noise algorithms and then later on performance of the algorithms in LE and DFE cases is compared to each other.

The following simulation setup is used to judge the performance.

- The average MSE is the performance parameter through which all the algorithms are analyzed.

- A $2 \times 2$ MIMO system is considered in this section

- $\quad$ SNR is kept at $20 \mathrm{~dB}$ for 10 and 20 users

- AWGN channel environment

- $\quad$ BPSK and QPSK modulations

Simulation results for comparison of the convergence speed of all algorithms for 10 and 20 users, in an AWGN channel, are shown, in figures. 2 and 3 respectively. As evident from both figures, this algorithm is converging faster than its competitors. It is also noted that MSE degenerates as the number of users is increased from 10 to 20 . When number of users is $10, \mathrm{MN}$ CLMS algorithm for LE case achieved MSE at $-6 \mathrm{~dB}$ in 120 iterations whereas the very first of other algorithms converged at MSE at $-6 \mathrm{~dB}$ in 140 symbols, MNCLMS algorithm achieved MSE at $-2.6 \mathrm{~dB}$ and in 140 symbols when number of users is doubled.

The MNCLMS algorithm's performance is evaluated in the AWGN environment using. The result in figure 6 shows the 
(IJACSA) International Journal of Advanced Computer Science and Applications,

TABLE I. LE COMPUTATIONAL COST PER ITERATION FOR DIFFERENT ALGORITHMS

\begin{tabular}{|c|c|c|}
\hline Algorithm & No. of Multiplications & No. of Additions \\
\hline \hline LMS & $2 K+1$ & $K$ \\
\hline RLS & $K^{2}+5 K+1$ & $K^{2}+3 K$ \\
\hline MNCLMS[12] & $2 K+1$ & $2 K+6$ \\
\hline MIMO-MNCLMS(LE)[9] & $2 M K+8$ & $2 M K+4$ \\
\hline
\end{tabular}

TABLE II. DFE COMPUTATIONAL COST PER ITERATION FOR DIFFERENT ALGORITHMS

\begin{tabular}{|c|c|c|}
\hline Algorithm & No. of Multiplications & No. of Additions \\
\hline \hline LMS & $2 K+1$ & $2 K$ \\
\hline RLS & $K^{2}+5 K+1$ & $K^{2}+3 K$ \\
\hline MNCLMS[12] & $2 K+1$ & $2 K+6$ \\
\hline MIMO-MNCLMS(DFE)[7] & $2(M K+N Q)+8$ & $M K+N Q+4$ \\
\hline
\end{tabular}

In both tables $K$ shows length of the filter.

performance using BPSK modulation. As evident, MNCLMS algorithm for LE case, is much superior than the LMS and the NLMS algorithms as MNCLMS converges faster. The MNCLMS algorithm converges on MSE of $-16 \mathrm{~dB}$ in 2000 symbols. A similar performance gain for MNCLMS can be seen using QPSK modulation as shown in figure 7. It is also evident that althoug there is a little deterioration in convergence rate but MNCLMS algorithm outclassed LMS and NLMS algorithms.

From simulation results, it is evident that althoug both have better performance than other constrained algorithms but when LE and DFE cases are compared with each other, it is evident that DFE is converging faster than the LE algorithm in the AWGN channel environment as in DFE case, the algorithm achieved MSE at $-16 \mathrm{~dB}$ as compared to LE which achieved MSE at $-6 \mathrm{~dB}$.

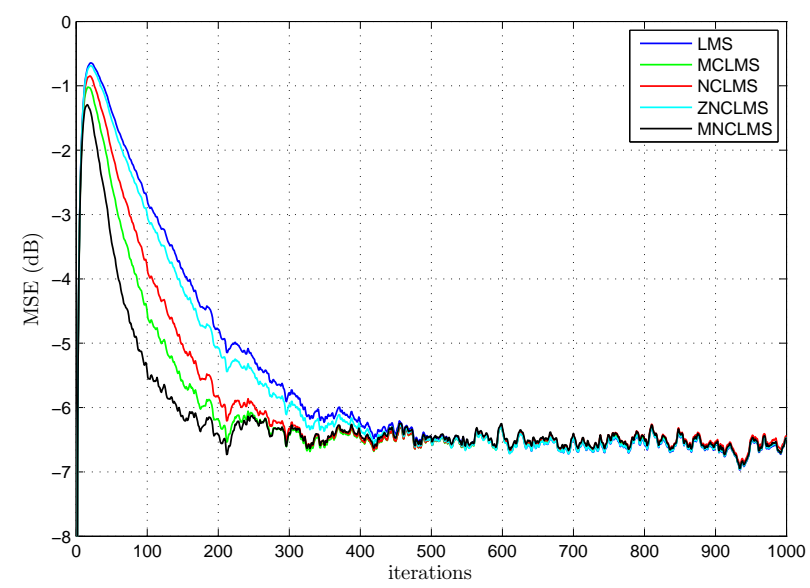

Fig. 2. Mean squared error (MSE) behavior of comparing algorithms in an AWGN channel environment for 10 users at $20 \mathrm{~dB}$ SNR

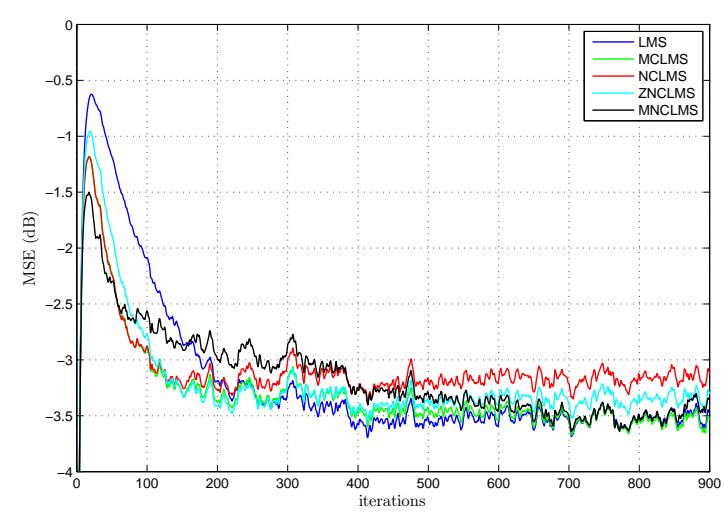

Fig. 3. Mean squared error (MSE) behavior of comparing algorithms in AWGN environment for 10 users at $20 \mathrm{~dB}$ SNR

Behavior of step size of MNCLMS algorithm in LE case is shown in figure 4 for 10 users. In the transient state algorithm in LE case is having the biggest step size value as compared to other algorithms and, thus, converges faster. In the steady state condition, step size parameter of this algorithm is minimized as compared to LMS, NCLMS and ZNCLMS algorithms. Same behavior is achieved for 20 users as shown in figure 5 .

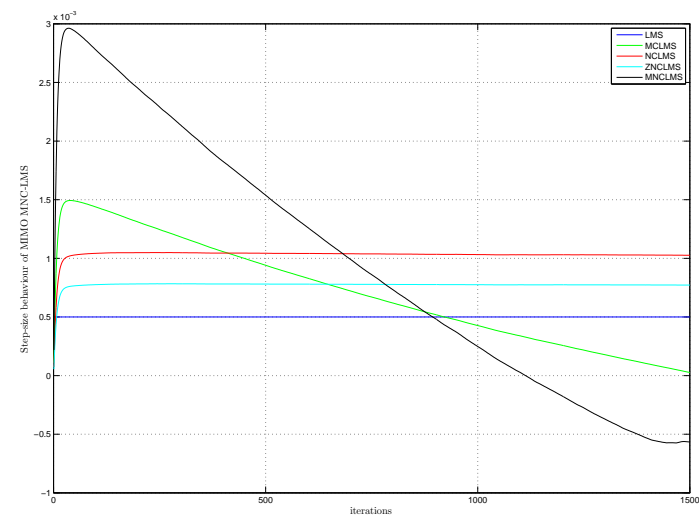

Fig. 4. Behavior of the step size of the LE algorithm for 10 users at $20 \mathrm{~dB}$ SNR 


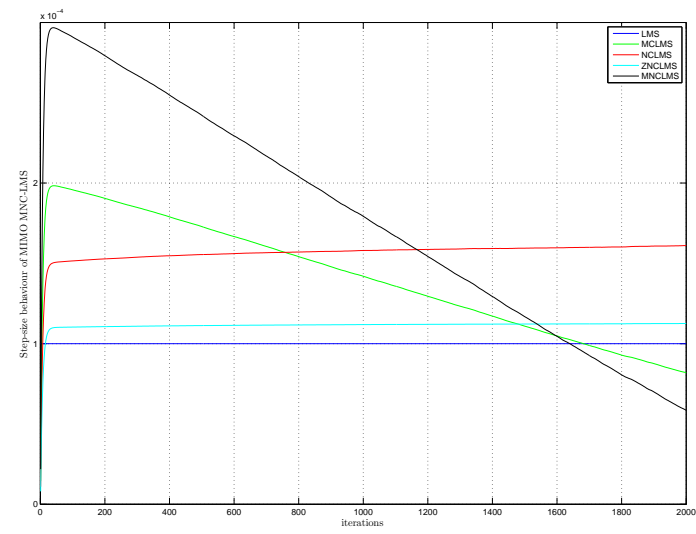

Fig. 5. Behavior of the step size of the MNCLMS algorithm for 25 users at $20 \mathrm{~dB}$ SNR

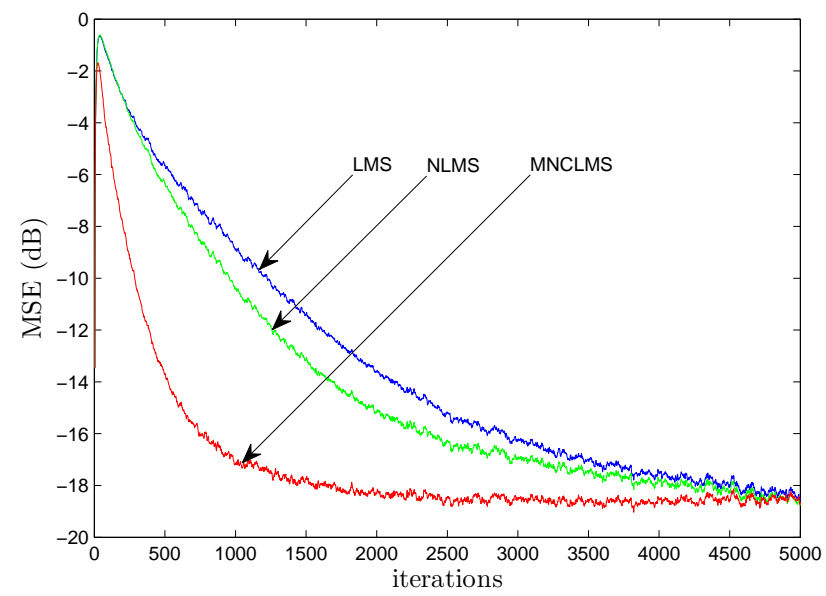

Fig. 6. Mean squared error (MSE) performance in AWGN channel environment at $\mathrm{SNR}=20 \mathrm{~dB}$

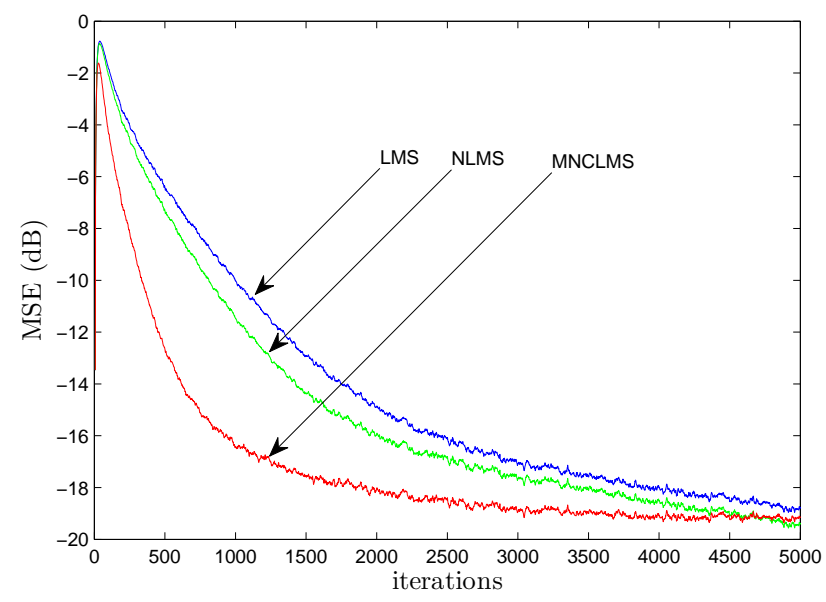

Fig. 7. Mean squared error (MSE) performance in AWGN environment at $\mathrm{SNR}=20 \mathrm{~dB}$ using QPSK signaling.

\section{B. Interference Elimination in Rayleigh Fading Channel for LE and DFE Cases}

Performance of the MNCLMS in the LE and DFE is compared to the standard LMS, MCLMS noise constrained LMS and ZNLMS algorithms in this section.

The following simulation setup is used to judge the performance of algorithms

- $2 \times 2$ MIMO system

- Random signature sequence having a length of 31 and a rectangular chip waveforms

- The SNR is kept at $10 \mathrm{~dB}$ and $20 \mathrm{~dB}$ for 10 and 20 users respectively

- Rayleigh fading channel environment

- $\quad$ BPSK and QPSK modulations

- $\quad$ Doppler frequency of $f_{d}=250 \mathrm{~Hz}$

Simulation results for convergence of the above mentioned algorithms for 10 and 20 users, in the Rayleigh fading channel are presented in figures 8 and 9. It can be seen that MIMO CDMA MNCLMS algorithm converged faster than its competitor algorithms. For 10 users, the algorithm achieved MSE at $-4.8 \mathrm{~dB}$ in 200 iterations. It is also observed that when number of users is enhanced to 20, the MNCLMS algorithm achieved MSE at $-5.2 \mathrm{~dB}$ in 240 iterations.

In second case, a flat fast Rayleigh fading channel is considered. As evident, form figure 11, MNCLMS in DFE case converged much earlier than its competitors. Figure 10 shows performance of the MNCLMS algorithm using QPSK modulation and as evident again, this algorithm converged much earlier than the LMS, NLMS etc.

It is also evident from the simulation results that MNCLMS algorithm in DFE case is converging much faster than the algorithm in LE case. As shown in figures 10 and 11, the algorithm for DFE case achieved MSE in $-16 \mathrm{~dB}$, which is much smaller than the algorithm in LE equalizer case.

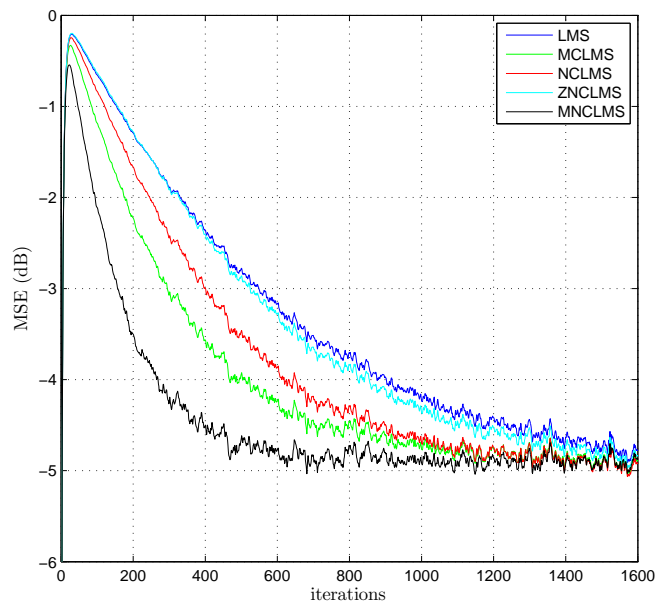

Fig. 8. Mean squared error (MSE) behavior for comparing algorithms in the Rayleigh fading environment for 10 users at $10 \mathrm{~dB}$ SNR 


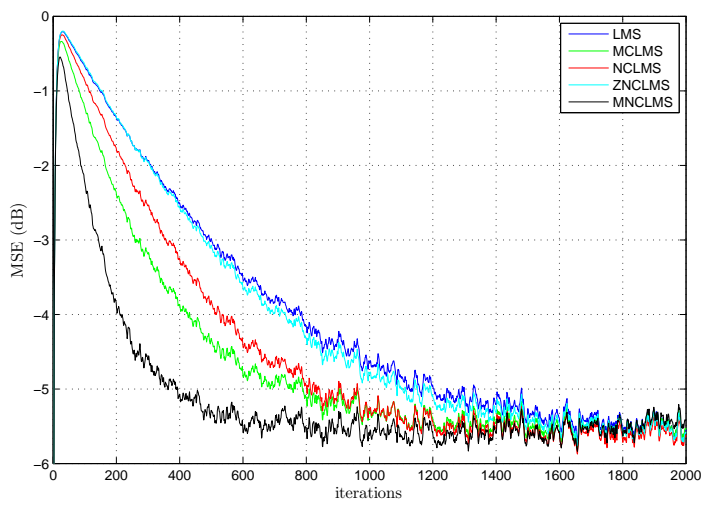

Fig. 9. Mean squared error (MSE) behavior for comparing algorithms in the Rayleigh fading environment for 20 users at $20 \mathrm{~dB}$ SNR

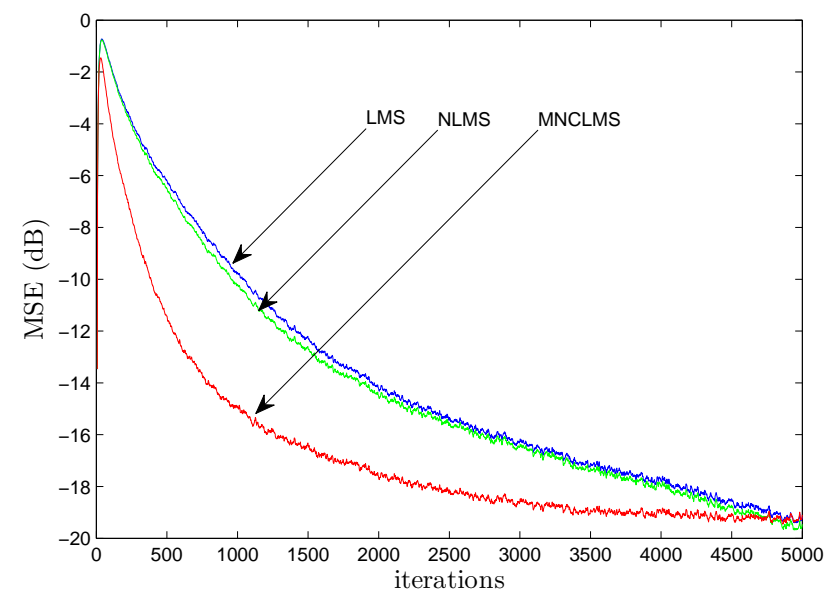

Fig. 10. Mean squared error (MSE) performance in Rayleigh fading environment with $f_{d}=250 \mathrm{~Hz}$ at $\mathrm{SNR}=20 \mathrm{~dB}$

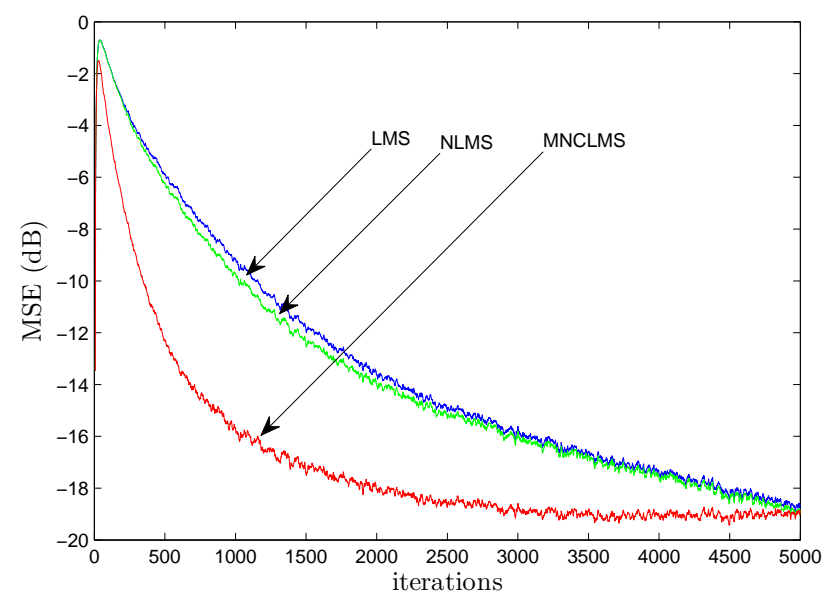

Fig. 11. Mean squared error (MSE) performance in Rayleigh fading environment with $f_{d}=250 \mathrm{~Hz}$ at $\mathrm{SNR}=20 \mathrm{~dB}$

\section{CONCLUSION}

A performance comparison between a MNCLMS algorithm for MIMO CDMA, DFE and LE cases is performed in this paper. Both algorithms are constrained on the length of spreading sequence, number of users, statistics (variances) of AWGN, MAI and additive noise. Simulation results are presented to compare the performance of the MNCLMS constrained algorithms in both cases and it is found that while both algorithms outperformed other constrained algorithms but when compared to each other, DFE has outperformed the LE.

\section{REFERENCES}

[1] Yongbin Wei, Saul B Gelfand, and James V Krogmeier. Noise constrained lms algorithm. In Acoustics, Speech, and Signal Processing, 1997. ICASSP-97., 1997 IEEE International Conference on, volume 3, pages 2353-2356. IEEE, 1997.

[2] RADU CIPRIAN Bilcu, Pauli Kuosmanen, and Corneliu Rusu. A noise constrained vs-lms algorithm. In EUROCOMM 2000. Information Systems for Enhanced Public Safety and Security. IEEE/AFCEA, pages 29-33. IEEE, 2000.

[3] Yongbin Wei, Saul B Gelfand, and James V Krogmeier. Noiseconstrained least mean squares algorithm. Signal Processing, IEEE Transactions on, 49(9):1961-1970, 2001.

[4] SONG Woo-Jin. A complementary pair $1 \mathrm{~ms}$ algorithm for adaptive filtering. IEICE TRANSACTIONS on Fundamentals of Electronics, Communications and Computer Sciences, 81(7):1493-1497, 1998.

[5] SONG Woo-Jin. A complementary pair $1 \mathrm{~ms}$ algorithm for adaptive filtering. IEICE TRANSACTIONS on Fundamentals of Electronics, Communications and Computer Sciences, 81(7):1493-1497, 1998.

[6] M Moinuddin, A Zerguine, and Asrar U. H. Sheikh. Multiple-Access Interference Plus Noise-Constrained Least Mean Square (MNCLMS) Algorithm for CDMA Systems. IEEE Transactions on Circuits and Systems I: Regular Papers, 55(9):2870-2883, October 2008.

[7] Khalid Mahmood, Syed Muhammad Asad, and Muhammad Moinuddin. Design of MAI Constrained Decision Feedback Equalizer for MIMO CDMA System. Technology.

[8] Khalid Mahmood, Syed Muhammad Asad, Muhammad Moinuddin, and Shashi Paul. Design of MAI Constrained Decision Feedback Equalizer for MIMO CDMA System. 2011.

[9] Khalid Mahmood, Syed Muhammad Asad, Muhammad Moinuddin, and Waqas Imtiaz. Mai and noise constrained lms algorithm for mimo cdma linear equalizer. International Journal of Advanced Computer Science \& Applications, 1(7):702-711, Jan 2016.

[10] A.H. Sayed and T. Kailath. A state-space approach to adaptive rls filtering. Signal Processing Magazine, IEEE, 11(3):18-60, 1994.

[11] Khalid Mahmood, Syed Muhammad Asad, Muhammad Moinuddin, and Shashi Paul. Design of MAI constrained decision feedback equalizer for MIMO CDMA system. 2011 International Conference on Wireless Communications and Signal Processing (WCSP), pages 1-5, November 2011.

[12] Muhammad Moinuddin, Azzedine Zerguine, and A Sheikh. Multipleaccess interference plus noise-constrained least mean square (mnclms) algorithm for cdma systems. Circuits and Systems I: Regular Papers, IEEE Transactions on, 55(9):2870-2883, 2008.

[13] Ali H Sayed. Adaptive filters. Wiley. com, 2008.

[14] a.H. Sayed and H.V. Poor. Optimal Multiband Joint Detection for Spectrum Sensing in Cognitive Radio Networks. IEEE Transactions on Signal Processing, 57(3):1128-1140, March 2009. 